\section{S. aureus nasel carriage among homosexual men with and without HIV infection}

To the Editor:

Staphylococcus aureus bacteremias have been recognized as a frequent complication of acquired immunodeficiency syndrome (AIDS) ${ }^{1-3}$ Several factors are thought to play a role in predisposing AIDS patients to clinical infections with this pathogen. ${ }^{4-6} \mathrm{We}$ and others have reported a high rate of $S$. aureus nasal carriage rate in hospitalized patients with AIDS ${ }^{2-9}$ Nasal carriage correlates with widespread skin colonization, ${ }^{10}$ which is considered in turn to be a risk factor for $S$. aureus infections, as it has been shown in patients undergoing long-term dialysis ${ }^{11,12}$ and in intravenous drug users. ${ }^{13}$

It is not readily apparent why patients with AIDS who are not intravenous drug abusers have an increased rate of $S$. aureus skin carriage. Our study population with AIDS consisted of hospitalized homosexual men, as we excluded all patients belonging to categories known to have an increased $S$. aureus carriage rate, particularly intravenous drug abusers. ${ }^{14-18}$ Persistent skin carriage of $S$. aureus is thought to result from frequent skin breaks through which the organism can penetrate and then spread throughout the skin, with the nose acting as a natural reservoir for skin colonization..$^{10}$ We conducted a controlled study to evaluate the influence of anal sex, with its attendant risk of mucosal and skin breakings,$^{19}$ on carriage rate of $S$. aureus and to assess the prevalence of $S$. aureus nasal carriage in an outpatient population of homosexual men in various stages of HIV (human immunodeficiency virus) disease.

Consecutive outpatients referred for HIV testing, and for follow-up of HIV infection or other sexually transmitted diseases were given a self-administered anonymous questionnaire on current sexual habits This included specific questions on anal intercourse (receptive versus insertive or both). For each person, cultures were obtained once from the anterior nares by means of sterile swabs (Culturette, Marion Scientific Co., Kansas City, Mo.). Specimens were cultured aerobically on blood agar, and S. aureus was identified by standard methods as previously described. The study was approved by the Institutional Review Board of the Foundation for Research on Sexually Transmitted Diseases. Informed consent was obtained from each participating subject.

Manifestations of HIV disease (classified according to the Centers for Disease Control [CDC] criteria), ${ }^{20}$ medical history, and use of antibiotics were reviewed. Patients with recent intravenous drug use (within 1 week), ${ }^{11}$ patients with insulin-requiring di-
Table 1. Nasal carriage of Staphyfococcus aureus in gay men according to stage of HIV infection and sexual habits ( $N$ )

\begin{tabular}{lcc}
\multicolumn{1}{c}{ Stage } & Total & $\begin{array}{c}\text { S. aureus } \\
\text { carriers }\end{array}$ \\
\hline AIDS/ARC & 65 & $21 / 65(32 \%)$ \\
Receptive anal sex & 46 & $16 / 46(35 \%)$ \\
HIV ( I) asymptomatic & 25 & $6 / 25(32 \%)$ \\
Receptive anal sex & 17 & $4 / 17(24 \%)$ \\
HIV $(-)$ & 39 & $7 / 39(18 \%)$ \\
Receptive anal sex & 20 & $4 / 20(20 \%)$ \\
Total & 129 & $36 / 129(28 \%)$ \\
Receptive anal sex & $83 / 126 *$ & $24 / 83(29 \%)$ \\
\hline
\end{tabular}

*Three subjects did not answer the questionnaire

abetes mellitus, and patients with chronic renal failure who were undergoing dialysis were excluded. Statistical analysis was performed by means of the $\chi^{2}$ test with Yates' continuity correction and with $95 \%$ confidence intervals to assess differences between groups.

All of the eligible persons consented to the study. Of 129 homosexual men, 65 had full-blown AIDS or other manifestations of advanced HIV infection (CDC class IV), 25 were symptom-free HIVseropositive (CDC classes II and III), and 39 were HIV-seronegative, $S$. aureus nasal carriage was detected in $21(32 \%), 8(32 \%)$, and $7(19 \%)$ patients, respectively, from these groups. The rate of $S$. aureus carriage in the overall population was $28 \%$. The rate of $S$. aureus carriage among all the HIVseropositive patients, including those with AIDS (29/90 ur $32 \%$ ), was increased as compared with the HIV-seronegative ones $(7 / 39$ or $19 \%)$ and was close to statistical significance $(0.05<p<0.1,95 \% \mathrm{con}$ fidence interval for the difference $=-0.01$ to 0.29 ) .

Of 126 homosexual men who completed the questionnaire on sex habits, 98 reported that they practiced either type of anal sex: $27 \%$ of them were $S$. aureus carriers as compared to $28 \%$ of 28 who reported that they did not currently practice anal intercourse. When they were subdivided into those practicing receptive anal intercourse versus those practicing only insertive anal intercourse (thought to be less traumatic), the rate of $S$. aureus carriage remained $28 \%$ in both groups, which was the overall rate of carriage in the study group. Distribution of receptive anal sex was not skewed in favor of any specific category of HIV infection (Table 1).

None of the persons in any group had been hos- 
pitalized within the previous month. Twelve patients with AIDS or AIDS-related complex had been hospitalized more than 1 month before entry into the study; $25 \%$ of them were nasal carriers of $S$. aureus, as compared with $28 \%$ of the remaining subjects. S. aureus nasal carriage was present in $3(18 \%)$ of 17 subjects who were taking antibiotics active against staphylococci and in $32(28 \%)$ of 112 who were not ( $p=$ NS, 95\% confidence interval for the difference $=-0.30$ to 0.09$)$. Twelve $(27 \%)$ of 44 subjects who were taking zidovudine (known to have activity against gram-negative organisms) ${ }^{21}$ were found to be nasal carriers of $S$. aureus, as compared with $24(28 \%)$ of 85 who were not taking it.

A small group of heterosexuals, not including active intravenous drug users, was also tested: S. aureus nasal carriage was found in 5 of 22 persons with AIDS or AIDs-related complex (23\%) and in 2 of 12 symptom-free HIV-seropositive persons (17\%).

We did not find any evidence to suggest that anal sex predisposes to $S$. aureus carriage among homosexual men, and our epidemiologic survey shows that receptive anal sex is not per se a risk factor for persistent skin colonization with $S$. aureus. These data must be taken with caution, as they were not based on objective observation but, rather, on anonymous self-reporting.

On the other hand, we noted a difference in nasal carriage rate of $S$. aureus in relation to HIV infection, although not of the magnitude previously reported. ${ }^{6-8}$ The difference in carriage rate between subjects with HIV infection and the HIVseronegative subjects was not largc and could not reach statistical significance, probably because of the small number of HIV-seronegative subjects (only 39). Still, close to one third of our HIV-infected subjects were carriers of $S$. aureus in the nares. This rate was comparable to that of other patients previously reported to be frequent carriers of $S$. aureus, such as intravenous drug users ${ }^{14}$ and diabetic patients taking insulin. ${ }^{16}$ However, in our previous series on hospitalized AIDS patients, we found a carriage rate of $55 \%$, and others have reported a similar rate. ${ }^{8}$ In that study we used the same technique for nasal culture and investigated patients within 24 hours of admission. Those patients were likely to be sicker and to experience more complications related to their underlying disease than the present outpatient population. Some of them had long-term indwelling venous catheters, which have been reported to be associated with $S$. aureus infections. ${ }^{13}$ The patients may have also been contaminated with $S$. aureus from the hospital environment during the first 24 hours of admission. A similar phenomenon was described in patients admitted to the medical intensive care unit $(22 \%$ respiratory tract colonization rate with gram-negative bacilli within 24 hours from ad- mission). ${ }^{22}$ In our opinion, these factors may have created more favorable conditions for the acquisition of $S$. aureus skin carriage and may explain the different carriage rate observed in the two studies.

We did not find that the use of zidovudine had any influence on nasal carriage of $S$. aureus, but a trend toward decreased carriage rate in antibiotic users was noted. The influence of recent hospitalization could not be reliably analyzed; only a few patients had been hospitalized previously, and all these admissions had occurred more than 1 month before nasal culture.

Overall, it seems that HIV infection is capable of increasing the carriage rate of $S$. aureus, although it is not clear through which mechanism this phenomenon occurs. HIV-infected persons have been found to have impaired neutrophil and monocyte functions, ${ }^{4-6}$ and decreased mucosal IgA secretion..$^{23}$ Whether these factors could play a role in mucosal or cutaneous carriage of $S$. aureus is not known at present. If they are proved to be significant, one may suspect that more advanced stages of HIV disease could produce even more sustained defects of mucocutaneous immunity and a higher carriage rate of $S$. aureus. Skin carriage of $S$. aureus is known to be a risk factor for subsequent development of $S$. aureus infections in certain categories of patients. ${ }^{11 \cdot 13}$ However, the magnitude of this risk has never been formally assessed in HIV-infected persons. We think it should be properly tested and, if found to be significant, measures to eradicate $S$. aureus carriage should be studied.

Ruggero Battan, MD
Mario C. Raviglione, MD
Joyce Wallace, MD*
Susannah Cort, MD*
John F. Boyle, PhD
Angelo Taranta, MD
Cabrini Medical Center, New York, NY, and
"Foundation for Research on Sexually Trans-
mitted Diseases. New York, NY.
Reprint requests: Dr. Ruggero Battan, Division
of Endocrinology and Diabetes, University of
Massachusetts Medical Center, Worcester, MA
01605.

\section{References}

1. Jacobson MA, Gellermann H, Chambers H. Staphylococcus aureus bacteremia and recurrent staphylococcal infection in patients with acquired immunodeficiency syndrome and AIDS-related complex. Am J Med 1988; 85:172-6.

2. Whimbley E, Gold JW, Polsky B, et al. Bacteremia and fungemia in patients with the acquired immunodeficiency syndrome. Ann Intern Med 1986;104:511-4.

3. Raviglione MC, Battan R, Pablos-Mendez A, Aceves- 
Casillas $\mathbf{P}$, Mullen MP, Taranta A. Infections associated with Hickman catheters in patients with acquired immunodeficiency syndrome. Am J Med 1989; 86:780-6.

4. Nielsen H, Kharazmi A, Faber V. Blood monocyte and neutrophil functions in the acquired immunodeficiency syndrome. Scand J Immunol 1986;24:291-6.

5. Murphy PM, Clifford Lane H, Fauci AS, Gallin JI. Impairment of neutrophil bactericidal capacity in patients with AIDS. J Infect Dis 1988;158;627-30.

6. Ellis M, Gupta $S$, Galant $S$, et al. Impaired neutrophil function in patients with AIDS or AIDS-related complex: a comprehensive evaluation. J Infect Dis 1988; 158:1268-76

7. Raviglione MC, Mariuz P, Pablos-Mendez A, Battan R, Ottuso P, Taranta A. High Staphylococcus aureus nasal carriage in patients with acquired immunodeficiency syndrome or AIDS-related complex. AM I INFFCT CONTROL 1990;18:64-9.

8. Ganesh R, Castle D, McGibbon D, Phillips I, Bradbeer C. Staphylococcal carriage and HIV infection [Letter]. Lancet 1989:2:558.

9. Berger TG, Jacobson MA, Becker B, Chambers HF. Nasal carriage rate of Staphylococcus aureus (SA) in AIDS and ARC patients [Abstract 372]. Program and Abstracts. Twenty-ninth Interscience Conference on Antimicrobial Agents and Chemotherapy, Houston, TX, October 1989:163.

10. Sheagren JN. Staphylococcus aureus: the persistent pathogen. N Engl J Med 1984;310:1368-73, 1437-42.

11. Yu VL, Goetz. A, Wagener $M$, et al. Staphylococcus aureus nasal carriage and infection in patients on hemodialysis: efficacy of antibiotic prophylaxis. $\mathrm{N}$ Engl $\mathrm{J}$ Med 1986;315:91-6.

12. Luzar MA, Coles GA, Faller B, et al. Staphylococcus aureus nasal carriage and infection in patients on continuous ambulatory peritoneal dialysis. N Engl J Med 1990;322:505-9.

13. Tuazon CU, Sheagren JN. Staphylococcal endocarditis in parenteral drug abusers: source of the organism. Ann Intern Med 1975;82:788-90.

14. Tuazon CU, Sheagren JN. Increased rate of carriage of Staphylococcus aureus among narcotic addicts, I Infect Dis 1974; 129:725-7.

15. Smith JA, Connor JJ, Willis AT, Nasal carriage of Staph ylococcus aureus in diabetes mellitus. Lancet 1966;2: 776-7.

16. Tuazon CU, Perez A, Kishaba T, Sheagren JN. Staphvlococcus aureus carriage rate among insulin-injecting diabetic patients: an increased carrier rate. JAMA 1975;231:1272.

17. Kirmani N, Tuazon CU, Murray HW, Parrish AE, Shcagren IN. Staphylococcus aureus carriage rate of patients receiving long-term hemodialysis. Arch Intern Med 1978;138:1657-9.

18. Kirmani N, Tuazon CU, Alling D, Carriage rate of Staphylococcus aureus among patients receiving allergy in jections. Ann Allergy 1980;45:235-7

19. Reiner NE, Judson FN, Bond WW, Francis DP, Petersen NJ. Asymptomatic rectal mucosa lesions and hepatitis $B$ surface antigen at sites of sexual contact in homosexual men with persistent hepatitis $B$ virus infection evidence for de facto parenteral transmission. Ann Intern Med 1984;96:170-3.

20. Centers for Disease Control. Classification system for human T-lymphotropic virus type III/lymphadenopathy-associated virus infections. MMWR 1986;35:334-9

21. Elwell LP, Ferone R, Freeman GA, et al. Antibacterial activity and mechanism of action of $3^{\prime}$-azido-3: deoxythymidine (BW A509I). Antimicrob Agents Che mother $1987 ; 31: 274-80$

22. Johanson WG, Pierce AK, Sanford JP, Thomas GD. Nos ocomial respiratory infections with gram-negative bacilli: the significance of colonization of the respiratory tract. Ann Intern Med 1972;77:701-6.

23. Kotler DP, Scholes JV, Tiemey AR. Intestinal plasma cell alterations inacquired immunodeficiency syndrome. Dig Dis Sei 1987;32:129*38. 